\title{
Transforming Healthcare system with Blockchain
}

\author{
Monika Rani ${ }^{1}$, Piyush Verma ${ }^{2}$, Shivam Kumar ${ }^{3}$, Nikita Tayal ${ }^{4}$, Sahil Pant ${ }^{5}$ \\ * Assistant Professor, Department of Computer Science and Information Technology, Krishna Engineering \\ College Mohan Nagar Ghaziabad Uttar Pradesh, India \\ 22,3,4,5 Student, Department of Computer Science and Information Technology, Krishna Engineering College \\ Mohan Nagar Ghaziabad Uttar Pradesh, India
}

\begin{abstract}
Article Info

Volume 7, Issue 4

Page Number : 613-620

\section{Publication Issue :}

July-August-2021

\section{Article History}

Accepted : 01 Aug 2021

Published : 20 Aug 2021

Blockchain technology has been used in many industries providing security and preventing fraud so it can be beneficial in the medical industry too. One of the most essential blockchain features that can be implemented in the healthcare sector is that by recording every change across the peer-to-peer network, every transaction is validated. Since clinical trials require fast, reliable and transparent ways to locate patient's data, blockchain could be the possible solution. Effective supply chain management is a challenge in every sector but in healthcare, it comes with added complexity and risk as it is related with the patient's health and safety. Effective management of the healthcare supply chain (HCSC) process is critical for healthcare providers not only during pandemics such as COVID-19 but also in their normal operations. Despite many advances in new technologies and treatment options, the providers still suffer from poor procurement, ordering, forecasting, and distribution practices. Hence, blockchain can be the potential solution for the security, integrity and functionality of health supply chain.
\end{abstract}

Keywords : Healthcare Supply Chain, COVID-19, Blockchain Technology

\section{INTRODUCTION}

It is necessary for the demand of revolution in technology in every sector. The healthcare sector also needs to make the most of the changes and equip itself with the best services and facilities it can provide. Blockchain can revolutionize healthcare and can change how patient's data is accessed by the physicians, how clinical research will take place and other aspects of the healthcare system. Blockchain has the capability to serve as a trusted, secure and interoperable component for effective and transparent healthcare ecosystem, both regionally and globally. In existing system, hospitals and health systems are require to enter the patient's data manually across multiple systems including the patient ID. This process can be automated using blockchain, once a physician enters the details and searches for potential patients, it can easily be mapped and with the right identifiers, matches of the right 
people can be found faster with improved accuracy and reliability. In particular, healthcare supply chains (HCSC) can be beneficial in providing significant improvements to healthcare providers ranging from cost savings, trusted and secure system, efficient and improved treatment outcomes to enhance patient safety and service quality [2].

\section{PRESENT CONDITION}

- Drug Counterfeit: counterfeit medicines are harmful as either they don't contain the correct ingredients in the right amount or contain entirely different ingredients that are not required and are ineffective and can cause side effects.Healthcare industry not only loses millions of dollars of revenue but also has a huge impact on the patient. Hence, traceability of the drugs becomes of the utmost importance.

- Clinical Trials: It is a method of testing a new drug and its effectiveness and side effects in a controlled environment which can take years to complete.The pharmaceutical companies need to invest heavily in trials and put a lot at stake for the companies, hence there is no doubt that we see frauds in clinical trials. There should be a transparent solution that allows anyone to review the clinical report and ensure that the results are not tampered.Clinical trials produces tons of data which includes statistics, reports, surveys, medical imagery, blood tests etc. which are to be made sure collected in the proper and secure manner. Fraud happens in the form of data manipulation. Some data is kept hidden, it doesn't reach to the system and some are tampered to reach the desired result.The critical information such as research protocol, hypothesis, data storage methods are not shared before the clinical trials which opens the opportunities for the modification of the data to reach the desired results.Also forms are mostly incomplete and hence, fabricated.
- Patient Data Management: As each patient's body reacts differently to the same disease, it is not necessary that the treatment that worked on one patient will work in the same manner for others.Hence, it is not possible to create a structure or use the same treatment strategy.Such complex issues makes it necessary to have the complete history of the patient's medical records. Hence, the patient data is needed to be stored securely and should be prevented from the leak while sharing through social media by the doctor.Therefore, a secure sharing and storing medium should be there for the management of the patient data.

- Claim and Billing: Fraudulent activities in healthcare billing and claims have become more popular and remain unnoticed with billing suffering the most. Hospitals usually carry the activities such as providing the bill to the patients with services that are either never taken by them or are overpriced than the industry standards. All such activities are done to maximize profit and keep the interest of the patients at bay. Not only that, hospitals and physicians are often caught many times improperly presenting the health condition of the patient which makes things even worse.While verifying the claims, the intermediaries involved are also reluctant in their job and leave the user hanging.The processing of the claims takes a lot of time and the user has to visit the office many times before it gets processed.

\section{Solution with Blockchain}

- Drug Traceability: Blockchain works by adding the transactions to the block. These transactions are unit immutable and also are time stamped for later verification. So, if the whole supply chain is moved to the blockchain, all of the issues can be fixed.Hybrid blockchain is the most appropriate 
solution with some aspects of its being private and some public.In this way the hospitals can keep their data safe as well as communicate with other systems and share public information.All drugs need to be registered on the blockchain before they began circulation. SSo, if a drug record isn't found on the blockchain, it will simply be deemed pretend and discarded from the provision chain. This straightforward methodology is accustomed to checking the believability of the medicine.Blockchain will give associate degree end-to-end resolution wherever platforms will work along. The systems will be able to track drugs and improve the full method of medicine square measure provided to the retailers and patients.

- Clinical Trials:As blockchain provides data integrity, it can be used as a proof for authentication of documents when needed to be verified.It also let's add new data which requires other nodes for the verifications process. Overall, the distributed networks make sure that data integrity is maintained and additionally confirm that no data are often changed while not licensed access.

- Solution for Patient data Management:The below figure illustrates the opportunities where blockchain could be employed to improve contracts in the healthcare environment. Smart contracts enable users to automatise and track bound state transitions. The party receiving new info receives an automatic notification and might verify the planned record before accepting or rejecting the info. This keeps participants educated and engaged within the evolution of the record. Overall, contracts are weakened into three branches: kinds of contracts offered to use, health pursuit knowledge, and method and storage.

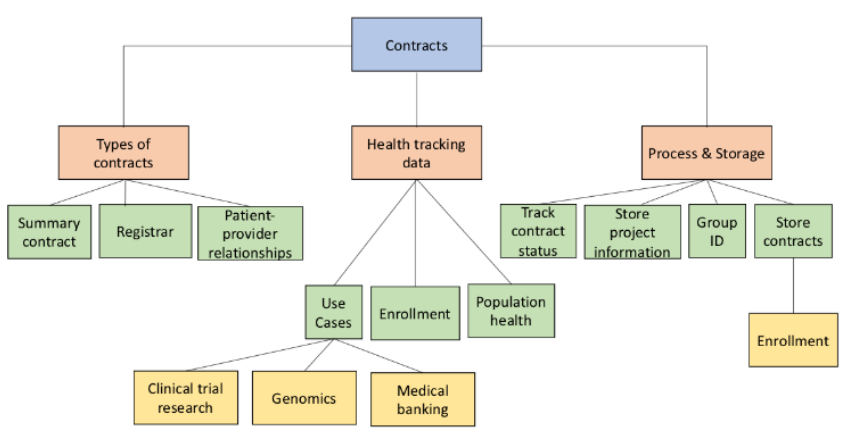

1. The first space of focus is the differing kinds of good contracts that permit users to change and track state transitions. The party receiving new data receives an automatic notification and might verify the planned record before accepting or rejecting the information whereas keeping the participants abreast of. The three varieties of good contracts are outline contracts, registrar contracts, and patient-provider relationship contracts [6].

2. An outline contract functions as a bread crumb path for participants within the system to find their medical history. Suppliers have references to patients they serve and third parties with whom their patients have licensed information sharing [6].

3. Good contracts permit taking parties to create a trusting relationship through hyperbolic transparency and pursuit with every transition and record evolution.

4. Smart contracts may advance ways used for pursuit of health information. As antecedently mentioned, cross-institutional information exchange will permit information to be gathered on patients on a microscopic level to the macroscopic level once comparing patients with similar conditions and/or demographics [7].

5. Good contracts may well be wont to track enrollment for health plans giving suppliers and patients an improved understanding of profit utilization [8].

6. In terms of population health, information gathered across the nation may well be accessed by researchers and establishments to check medical 
specialty once information (or tips to these data) are kept within the blockchain, creating the information practical, secure, and simply accessible and traceable. Good contracts permit taking parties to know the employment of health information whereas providing secure and auditable pursuit of access.

7. One important advantage of a sensible contract is its ability to produce method and storage for contracts, project management, and health information. Contracts and different important data are kept on the blockchain, establishing a network of trust among the parties concerned. This permits health organizations to share patient information that is identifiable, guaranteeing confidentiality. The localized info answer provided by blockchain could be a key resolution to ability and record storage whereas maintaining health information and different health-related data [9].

\section{GPO:-}

Effectively managing the tending provide chain (HCSC) method is crucial for tending suppliers not solely throughout pandemics like COVID-19 however additionally in their traditional operations. Despite important advances in new technologies and treatment choices suppliers still suffer from poor acquisition, ordering, statement, and distribution practices. cluster buying Organizations (GPOs) square measure a very important neutral in HCSC and profit suppliers with value savings, volume discounts, and vendor choice. However, the present United States Government Printing Office contract method is long and lacks potency. Hence, our projected answer integrates blockchain technology and decentralized storage to push transparency, streamlines communication with stakeholders, and minimize the acquisition timeline while avoiding evaluation discrepancies and inaccuracies. Our answer connects all the stakeholders like manufacturer, GPO, distributor, and supplier victimisation Ethereum network. During this paper, we tend to propose a blockchain answer victimisation sensible contracts to alter the United States Government Printing Office contract method. We tend to propose a generic framework for the catching method within the HCSC with elaborate algorithms portraying numerous interactions among HCSC stakeholders. The sensible contract code was developed and tested victimisation Remix IDE and also the code is publicly shared via Github. We tend to discuss numerous security risks and give elaborate analysis of varied transactions incurred by the stakeholders. Our analysis demonstrates that the projected blockchain-based answer is economically possible as solely a borderline group action fee is gone by the stakeholders within the distributed network.

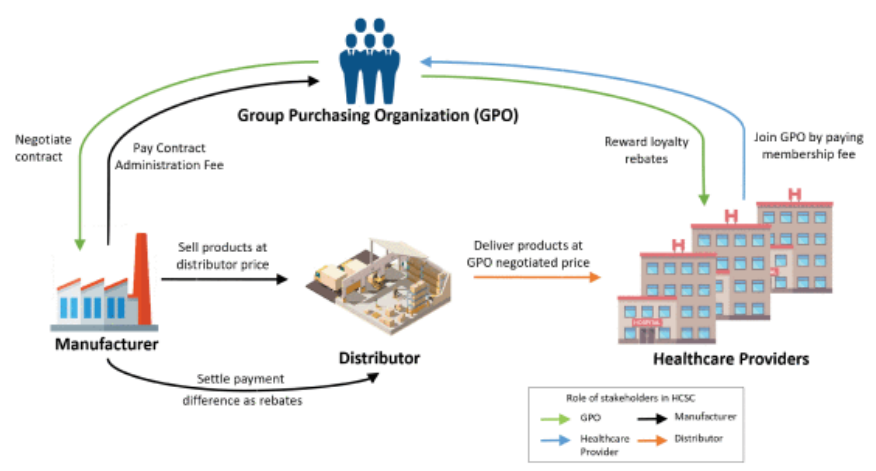

Figure 1. Current GPO Process

Proposed Blockchain Based GPO Contract Administration Solution :-

We propose a blockchain-based GPO contract solution that connects manufacturers, distributors, GPOs, and healthcare providers within the same decentralized Ethereum network, as illustrated in Fig. 2. Our solution adopts blockchain technology to promote transparency, data provenance, and data immutability in the contracting process. These stakeholders interact with one another using the smart contracts shown in Fig. 2. Furthermore, we describe the role of each stakeholder and component in our solution. 
- Manufacturer: A product or device producing firm that develops and sells medical instrumentality or medicine to be utilized by tending suppliers.

- Group buying Organization (GPO): a company specialised in serving to tending suppliers like hospitals, home health agencies, nursing homes, and ambulant care facilities. They combine buying volumes and use that leverage to barter marketing costs with makers to get discounts achieved by economies of scale [3]. Thus, suppliers like operating with GPOs as they gain buying power and have the benefit of value savings. GPOs don't purchase the products; instead, they negotiate contracts for a specific product with potential vendors on behalf of their suppliers supported the commitment that a minimum buying volume would be bought by the suppliers throughout the life of the contract.Providers become members of the US Government Printing Office by paying Associate in Nursing annual membership fee whereas makers collaborate with GPOs by paying contract administration fees (CAF) [4]. CAF may be a share of the entire dealing worth of product purchased within the negotiated contract [4]. Thus, the operational expenses of GPOs return from the CAF and also the membership fee paid by suppliers. In return, GPOs sometimes reward suppliers with atiny low share of the CAF as loyal rebates for future purchases.

- Distributor: generally, distributors square measure wholesalers placed between makers and tending suppliers. They obtain products from makers at a distributor worth, that is far on top of the contract worth united between the US Government Printing Office and manufacturer. Thus, the distinction between the distributor worth and also the contract worth is rescued by the distributor as rebates from the manufacturer once the distributor delivers product to suppliers registered within the US Government Printing Office contract.Moreover, distributors would sometimes ought to conduct sale tracings to assert rebates, however with blockchain technology, this tedious task would not be allotted manually as historical information of all valid transactions would be keep for good within the blockchain ledger. Likewise, makers wouldn't ought to verify the distributor's claims on whether or not the merchandise was oversubscribed to the supplier at the negotiated contract's worth or not. As a result, the distributor gains its ratio from delivery fees and manufacturer rebates.

- Healthcare Provider: tending suppliers like nursing homes and hospitals sometimes obtain products from distributors employing a US Government Printing Office contract or directly from the manufacturer while not having to travel through the US Government Printing Office and distributor as intermediaries. However, our resolution focuses on the previous technique of buying products. Therefore, once a contract is awarded to the manufacturer, then all suppliers registered within the contract should buy the required product a the negotiated united worth explicit within the contract. At the same time, the US Government Printing Office appoints a distributor that will link the suppliers to the manufacturer. Hence, permitting suppliers to send purchase orders on to the distributor exploitation the negotiated worth explicit within the contract.

- Decentralized Storage Technology: A distributed peer-to-peer filing system that helps in connecting an equivalent filing system to all or any nodes within the network. Their square measure varied file systems, like celestial body filing system (IPFS) or Filecoin. a giant amount of information is kept in IPFS by licensed users within the network. Then changeless IPFS links are created and kept in blockchain transactions as timestamped valid transactions secured exploitation scientific discipline technology. This is often helpful since stakeholders don't need to worry about storing an oversized quantity of information within the blockchain itself. Thereby creating the mix of distributed ledger and storage appropriate for US 
Government Printing Office contract resolution since solely the indexed info would be kept on the chain, which might find specifically wherever the info is kept within the IPFS.

- Ethereum sensible Contracts: Second-generation blockchain platforms like Ethereum permit codes to be written within the kind of sensible contracts. These contracts are programmed to execute functions mechanically while not interference from third parties. They act as package agents that make sure that all stakeholders within the network abide by the terms and conditions explicit within the contract.

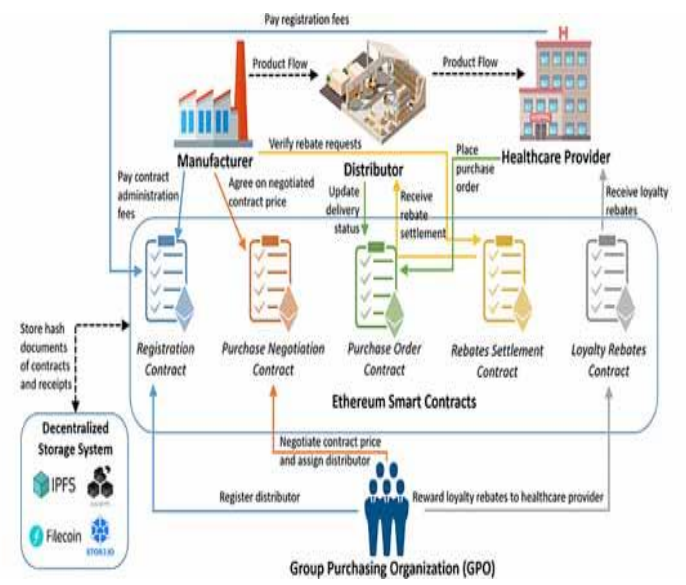

Figure 2: System overview of a blockchain-based GPO contract solution using Ethereum smart contracts and decentralized storage system.[1]

The system is captured as a series of functions and events in sequence diagrams in which the interaction between each stakeholder and contracts are captured, as shown in Fig. 3. Firstly, the system allows the GPO to deploy the Registration contract. Each stakeholder in the network is registered using their Ethereum address in the registration contract. The manufacturer and provider confirm their registration by paying an administration fee and an annual membership fee respectively by calling the registerManuf() and registerHP() functions. Then the GPO initiates a new pricing negotiation contract for a particular product or a group of products by calling the newContract function on behalf of the providers in the PriceNegotiation contract. The manufacturer negotiates the suggested GPO price by using the PriceNegotiation() function. The GPO confirms or rejects the suggested price through the contractStatus() function. When the contract is confirmed, then an event is triggered, notifying all stakeholders in the network using the ContractConfirmed() function. The successful contract is then uploaded in the decentralized storage system and published for registered providers to purchase using the negotiated contract price. Moreover, the GPO assigns a distributor between the manufacturer and provider to service the contract via assignDistributor() function. Then the stakeholders are notified on the successful completion of the contract agreement via ContractClosed() function. Then the registered provider is allowed to make a purchase order using the submitPO() function in the PurchaseOrder contract. The distributor delivers the order while providing the order status in the deliveryStatus() function. Once the order is successfully delivered, then an event is triggered using the orderDelivered() function.

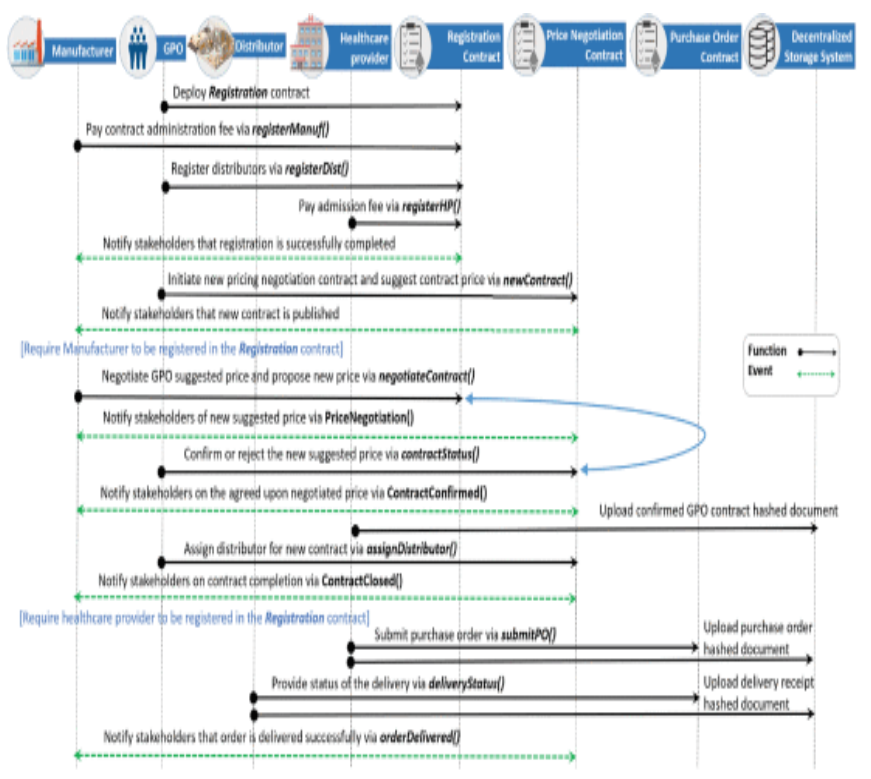

Figure 3. Sequence diagram showing the function calls and events between stakeholders when initiating GPO contract. 
Moreover, the sequence diagram shown in Fig. 4 captures the rebates settlement between manufacturer and distributor once the order is delivered with the successful RebateSettlement contract. The distributor submits a rebate request using the submitRebateRequest() function. The manufacturer then approves the request and transfers the required amount via the approveRebateRequest() function. This, in return, triggers an event notifying all stakeholders that the distributor received the rebate requested. On the other hand, the GPO transfers loyal rebates to providers as a reward for fulfilling the contract using the sendLoyaltyRebate() function. This would trigger an event to notify providers that the amount is transferred successfully using the LoyaltyRebateTransferred() function.

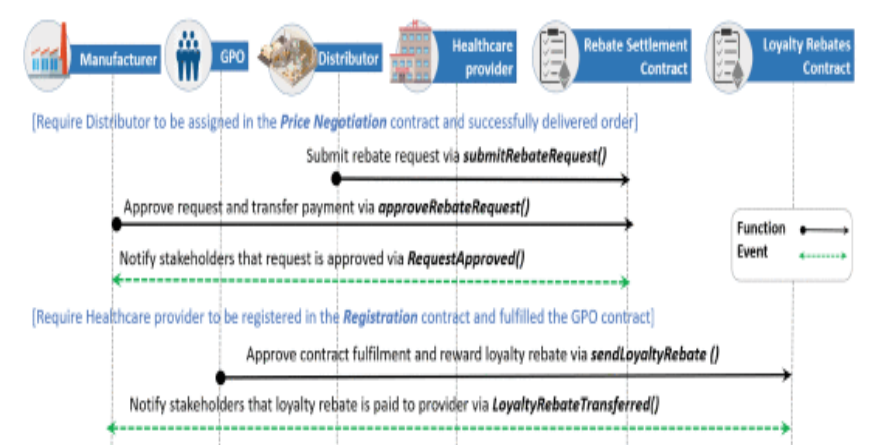

FIGURE 4. Sequence diagram showing the function calls and events between stakeholders when claiming rebates.

\section{FUTURE SCOPE}

As we look to take medical records from a research prototype to a meaningful tool for enterprise, government and patient use, we have identified several thrusts of future work. First, we continue our process of actively engaging with healthcare stakeholders across the industry, from hospitals and provider offices, to pharmaceutical companies, to insurance companies, to healthcare startups and Government institutions. In future months, we hope to complete additional rounds of security testing, including third-party penetration testing, as outlined in the ONC Roadmap's guidelines for "Ubiquitous, Secure Network Infrastructure" [10].

Currently, the cost of transaction on Ethereum Blockchain is too high so, while building the project one can use the second layer solution like "Polygon Network" for lowering the overall deployment and transaction costs.

After sometime a separate blockchain can be deployed for this special use case using Cosmos SDK and tedermint for the consensus mechanism where all the different stakeholders in the healthcare industry can become the node operators and can be the validators of the blockchain. This can be a permissioned blockchain where only the node operators will have the authority for accessing the data and thus further safeguarding the sensitive user information.

\section{CONCLUSION}

The blockchain technology is very promising for this particular use case where it is required to handle large-scale sensitive data in a secure manner as it uses various cryptographic techniques that strengthen the overall security of the network.

Blockchain has proven beneficial in the nonhealthcare sectors with an improved supply chain in terms of accountability, transparency and traceability and the same can be beneficial for the medical industry.

As Blockchain technology safeguards from drug counterfeiting, it can also be useful in vaccine distribution in a country like India where we have such a big population and blockchain can ensure the right amount of automation, transparency and security needed in the process of vaccination. 
We discussed how the presence of GPOs in HCSCs helps various stakeholders in the HCSC, in particular the providers by achieving cost savings from quantity discounts and operational savings due to efficient procurement practices. The proposed solution guarantees that only registered stakeholders are allowed to register and interact with the smart contract ensuring trust and transparency among stakeholders.

\section{REFERENCES}

[1]. I. A. Omar, R. Jayaraman, M. S. Debe, K. Salah, I. Yaqoob and M. Omar, "Automating Procurement Contracts in the Healthcare Supply Chain Using Blockchain Smart Contracts," in IEEE Access, vol. 9, pp. 3739737409, 2021, doi: 10.1109/ACCESS.2021.3062471.K”

[2]. R. Jayaraman, K. Taha, K. S. Park and J. Lee, "Impacts and role of group purchasing organization in healthcare supply chain", Proc. IIE Annual Conf., pp. 3842, 2014.

[3]. A. Ahmadi, M. S. Pishvaee and M. Heydari, "How group purchasing organizations influence healthcare-product supply chains? An analytical approach", J. Oper. Res. Soc., vol. 70, no. 2, pp. 280-293, Feb. 2019.

[4]. Q. Hu, L. B. Schwarz and N. A. Uhan, "The impact of group purchasing organizations on healthcare-product supply chains", Manuf. Service Oper. Manage, vol. 14, no. 1, pp. 7-23, Jan. 2012.

[5]. M. Gaynor, J. Tuttle-Newhall, J. Parker, A. Patel and C. Tang, "Adoption of blockchain in health care", J. Med. Internet Res., vol. 22, no. 9, Sep. 2020.

[6]. Ekblaw A. A Case Study for Blockchain in Healthcare: 'MedRec' Prototype for Electronic Health Records and Medical Research Data. Office of the National Coordinator for Health Information. 2016.
[7]. Peterson K. A Blockchain-Based Approach to Health Information Exchange Networks. Colleaga: Harnessing the Power of Collective Intelligence. 2016.

[8]. Culver K. Blockchain Technologies: A Whitepaper Discussing How the Claims Process Can Be Improved. The Blockchain Lab. 2016.

[9]. Blockchain: The Chain of Trust and its Potential to Transform Healthcare - Our Point of View. Office of the National Coordinator for Health Information. 2016.

[10]. Office of the National Coordinator for Health Information Technology (2015). Version 1.0. "Connecting Health and Care for the Nation: A shared nationwide interoperability roadmap."

[11]. Q. J. Hu and L. B. Schwarz, "Controversial role of GPOs in healthcare-product supply chains", Prod. Oper. Manage., vol. 20, no. 1, pp. 1-15, Jan. 2011.

[12]. F. Benoit and L. A. McWhorter, The Challenges and Opportunities of Contract Price Alignment in Healthcare, Nov. 2020.

[13]. T. Hisey and R. Jacoby, The Role of Distributors in the US Health Care Industry, Nov. 2019

\section{Cite this article as :}

Monika Rani, Piyush Verma, Shivam Kumar, Nikita Tayal, Sahil Pant, "Transforming Healthcare system with Blockchain", International Journal of Scientific Research in Computer Science, Engineering and Information Technology (IJSRCSEIT), ISSN : 24563307, Volume 7 Issue 4, pp. 613-620, July-August 2021. Available at

doi : https://doi.org/10.32628/CSEIT217434

Journal URL : https://ijsrcseit.com/CSEIT217434 